\title{
N-doped graphene: Polarization effects and structural properties
}

\author{
Hossein Ghorbanfekr-Kalashami, ${ }^{1}$ M. Neek-Amal, ${ }^{2,3,{ }^{*}}$ and F. M. Peeters ${ }^{1}$ \\ ${ }^{1}$ Department of Physics, University of Antwerp, Groenenborgerlaan 171, B-2020 Antwerpen, Belgium \\ ${ }^{2}$ Shahid Rajaee Teacher Training University, 16875-163, Lavizan, Tehran, Iran \\ ${ }^{3}$ Institute of Advanced Technologies, Shahid Rajaee Teacher Training University, Lavizan, Tehran, 16788, Iran
}

(Received 22 January 2016; revised manuscript received 16 April 2016; published 19 May 2016)

\begin{abstract}
The structural and mechanical properties of $\mathrm{N}$-doped graphene (NG) are investigated using reactive force field (ReaxFF) potentials in large-scale molecular dynamics simulations. We found that ripples, which are induced by the dopants, change the roughness of NG, which depends on the number of dopants and their local arrangement. For any doping ratio N/C, the NG becomes ferroelectric with a net dipole moment. The formation energy increases nonlinearly with N/C ratio, while the Young's modulus, tensile strength, and intrinsic strain decrease with the number of dopants. Our results for the structural deformation and the thermoelectricity of the NG sheet are in good agreement with recent experiments and $a b$ initio calculations.
\end{abstract}

DOI: 10.1103/PhysRevB.93.174112

\section{INTRODUCTION}

Graphene is a crystalline allotrope of carbon with a two-dimensional honeycomb lattice structure, which has $s p^{2}$ in-plane covalent bonds [1,2]. This atomic thin material has gained considerable attention in the past decade due to its exceptional physical properties [3-6]. Substitutional (N) doping is an effective way to intrinsically modify the electric, chemical, and mechanical properties of carbonbased materials. Chemical vapor deposition (CVD) and ammonia heat-treatment processes are two of the practical techniques for fabricating nitrogen-doped graphene sheets. The produced samples have been investigated by several characterization techniques, including tunneling microscopy and spectroscopy [7-10]. Because of the higher electronegativity of the nitrogen atoms, it leads to a significant charge redistribution. The $\mathrm{N}$ dopant also influences the spin density, which causes that region to be more chemically active. Such regions participate directly in catalytic reactions, such as oxygen reduction reactions as well as anchoring sites for metal nanoparticles used in catalytic reactions [11-13]. By doping with nitrogen, a band gap is opened and the Fermi level is shifted above the Dirac point [7,11] (more details about different nitrogen doping methods, characterization methods, and applications can be found in Refs. [8,11,14]).

A density functional theory study (DFT) has been conducted to determine the structure of a single nitrogen dopant on a hosted graphene sheet [10]. Zhao et al. [7] used first principles techniques to calculate the formation energy of different N-dopant configurations. Fujimoto et al. [15] and Rani et al. [16] investigated the effect of substitutional nitrogen doping on the electronic band structure and the bond lengths. These types of studies are limited to a few dopants within a relatively small unit cell. Consequently, it leads to a less realistic description of doping and their collective impact on the thermoelasticity of doped systems. Some studies used a classical molecular dynamics (MD) approach to investigate the mechanical response of a (doped) graphene sheet based on semiempirical potentials $[17,18]$. Despite all such theoretical

\footnotetext{
*neekamal@srttu.edu
}

and experimental works on N-doped graphene, the ferroelectric properties of such doped systems have yet to be discussed, and more detailed studies are urgently needed to elucidate the structural deformation of the $\mathrm{N}$-doped configurations within the graphene sheet and the corresponding charge-induced polarization.

Here, we investigate the structural deformation and mechanical properties of $\mathrm{N}$-doped graphene using large-scale molecular dynamics simulations. We show that the uniformly random nitrogen atoms inserted in graphene change the morphology of the system and, depending on the local arrangement of the nitrogen atoms, the roughness can be very different. The nitrogen impurities decrease the elastic modulus of graphene and the fracture threshold. We predict that nitrogen-doped graphene is a ferroelectric with a permanent dipole whose strength depends on the particular distribution of the nitrogen atoms.

\section{THE MODEL AND COMPUTATIONAL METHODS}

Our simulation box (that is periodically repeated) for pristine graphene contains 11200 carbon atoms. We randomly distributed $\mathrm{N}$ atoms (i.e., we used the Monte Carlo method) over graphene by substituting carbon atoms with nitrogen. Each site of the graphene sheet has an equal chance to be replaced with an $\mathrm{N}$ atom (see Fig. 1). When we randomly generate $\mathrm{N}$ atoms in the graphene sheet, there is a chance for the appearance of some nitrogen atoms that are bonded to each other, which results in nitrogen clusters. In Fig. 1(d) we show a typical nitrogen cluster. For each concentration of $\mathrm{N}$ atoms we generated a set of systems and made an average in order to obtain the different physical quantities. Typically, we generated five systems per concentration and we used a computation unit cell of dimension $18 \times 18 \mathrm{~nm}^{2}$.

In this way we produce various dopant configurations similar to those observed in experiments [7]. We apply periodic boundary conditions (PBCs) in both the $x$ and $y$ directions and use the reactive force field (ReaxFF) potential between the different atoms [19,20]. An NPT ensemble (a Nosé-Hoover thermostat and barostat are used [21,22]) is used to keep the temperature and pressure constant. The simulation time step is 0.1 fs. All calculations have been performed by 


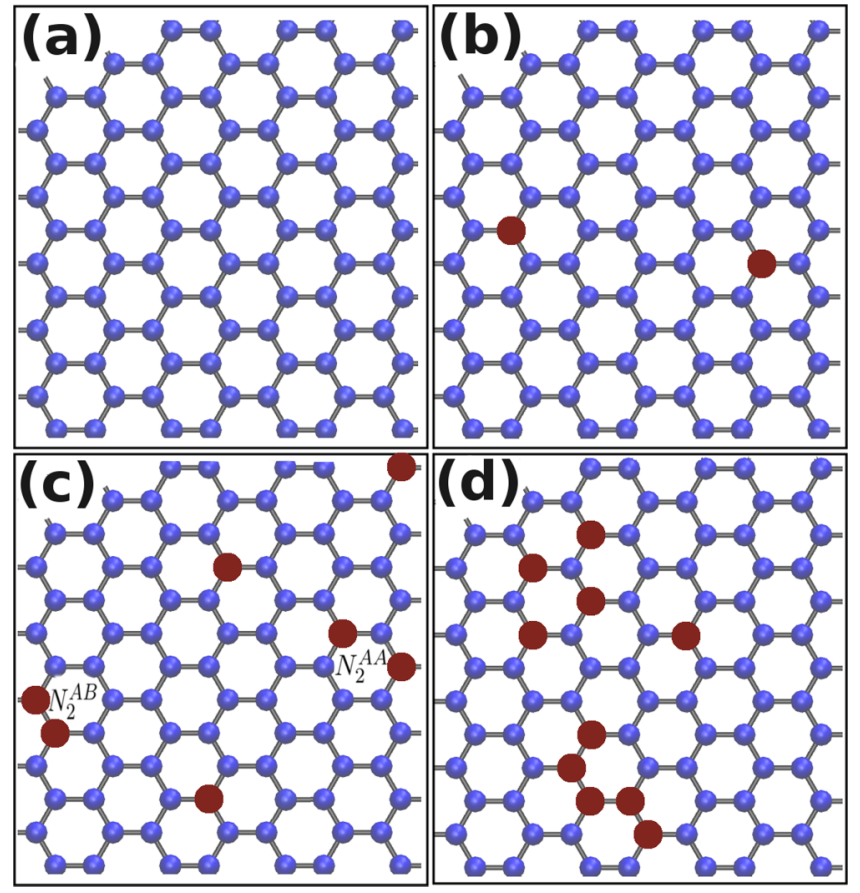

FIG. 1. (a) Pristine graphene, (b) graphene with $1 \%$ nitrogen, and (c) graphene with $3 \%$ nitrogen where double substitution of dopants $\mathrm{N}_{2}^{A A}\left(\mathrm{~N}_{2}^{A B}\right)$ is present within the same (different) nearest sublattice. (d) Graphene with $5 \%$ nitrogen where a single carbon (nitrogen) atom is connected to more than one nitrogen in the system (nitrogen clusters). The carbon and nitrogen atoms are shown as blue and brown (bigger) balls, respectively.

using the MD, large-scale atomic/molecular massively parallel simulator (LAMMPS) $[23,24]$.

Figure 1 shows a top view of some of the studied systems, i.e., pristine graphene [Fig. 1(a)] and doped graphene [Figs. 1(b)-1(d)] where some carbon atoms (blue balls) are randomly replaced with nitrogen atoms (brown balls) with, respectively, $1 \%, 3 \%$, and $5 \% \mathrm{~N}$ doping. Different local $\mathrm{N}$ configurations are possible, e.g., in Fig. 1(b) we depict single dopants which are surrounded by three carbon atoms, in Fig. 1(c) we show an example of the double substitution of dopants $\mathrm{N}_{2}^{A A}\left(\mathrm{~N}_{2}^{A B}\right)$ on the same (different) nearest sublattice, and in Fig. 1(d) a nitrogen cluster occurs, which is a single carbon (nitrogen) atom that is connected to more than one nitrogen. The studied systems resemble the experimental sample in Ref. [8], where several nitrogen species, such as pyridinelike, pyrrolelike, and quaternary nitrogen, have been formed. In our study, we dope the system up to $5 \%$, which covers the experimental concentration of $2.8 \%(5.6 \%)$ that was realized by the heat treatment of graphene under ammonia (atmospheric pressure CVD). They investigated experimentally the effect of nitrogen doping on the structural deformation and carrier transport of the graphene monolayer $[8,9]$.

To investigate the mechanical properties we relaxed the system for about $10 \mathrm{ps}$, and subsequently subjected it to an inplane tensile stress for $200 \mathrm{ps}$ which was realized by changing the length (width) of the system in the $x(y)$ direction using the displacement-control method described in Refs. [18,25]. The strain increment is applied every 5000 time steps with a rate value of $0.001 \mathrm{ps}^{-1}$. We set the $x(y)$ direction to be in the armchair (zigzag) direction. By calculating the stress tensor elements we are able to estimate the Young's modulus and the breaking stress (strain) of N-doped graphene.

\section{RESULTS AND DISCUSSION}

\section{A. Charge distribution and electric dipole}

First, we study the electric charge distribution over N-doped graphene (NG) at zero temperature. The density plot of the electric charge distribution on a system with $1 \%$ and $5 \%$ nitrogen is depicted in Figs. 2(a) and 2(b). It shows how nitrogen doping changes the local charge distribution and it leads to a (local) dipole in the graphene layer. To improve the quality of the pictures, the space between the atoms was
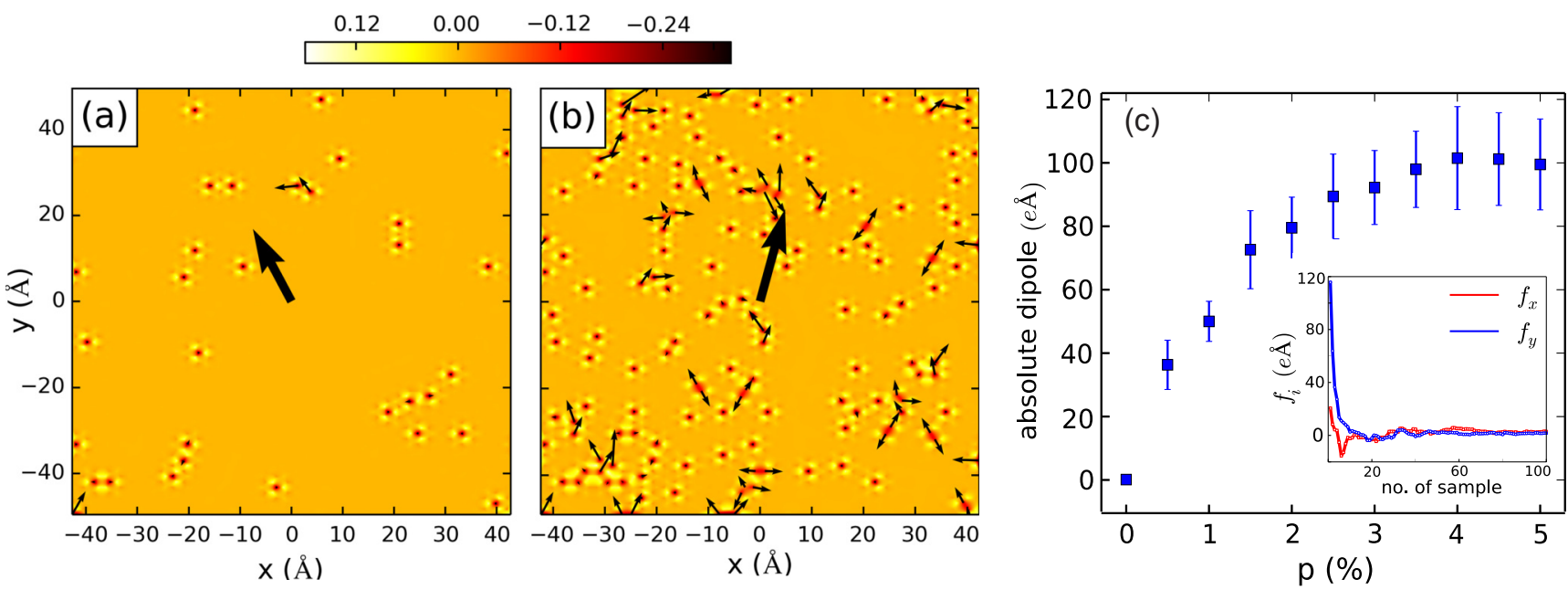

FIG. 2. Density plot of the charge distribution of doped graphene with (a) $1 \%$ and (b) $5 \%$ nitrogen doping. Small arrows indicate the local dipoles, and the thick arrow corresponds to the net dipole. The color scale is in units of the elementary charge. (c) Absolute value of the net dipole as function of the $\mathrm{N}$-dopant concentration. The inset indicates vanishing of the total dipole when we average over many samples, where $f_{x}$ and $f_{y}$ are the $x$ and $y$ components of the total dipole of $n_{s}$ samples. 

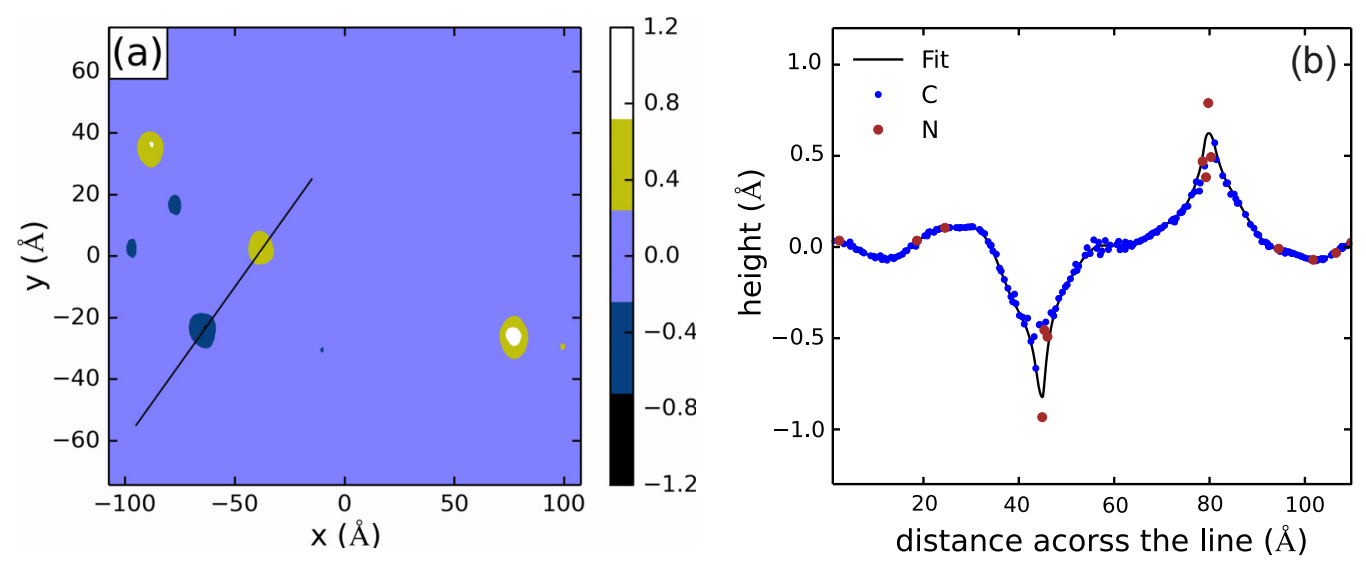

FIG. 3. (a) Height distribution of the NG atoms at room temperature for $5 \% \mathrm{~N}$ doping. The color scale is in units of $\AA$. (b) Height variation across the line in (a).

interpolated using a nearest-neighbor algorithm. When the nitrogen atoms are surrounded by three carbon atoms, the local dipole is zero, while for the other $\mathrm{N}$ configurations a local net electrical dipole appears (e.g., when two nitrogen atoms are linked). The total net dipole in each panel is shown by the thick arrow in the center of the picture. Note that the ensemble average of the net dipole should be zero, however, any individual sample has a net dipole with random orientation that depends on the local arrangement of the dopants (i.e., the presence of $\mathrm{N}$ clusters). In Fig. 2(c), we show the variation of the absolute value of the total dipole of the N-doped system with $p$. In fact, Fig. 2(c) represents the total absolute dipole moment of the system $|\vec{P}|$, where $\vec{P}=\sum_{i=1}^{\text {atoms }} q_{i} \vec{r}_{i}$ is averaged over five samples. The error bars shown in Fig. 2(c) correspond to the averaging over those five samples. In order to confirm that the net dipole is zero, we performed extra simulations and averaged over 100 samples, by defining $f_{i}\left(n_{s}\right)=\sum_{j=1}^{n_{s}} P_{j, i} / n_{s}$, where $i$ refers to $x$ or $y$ and $n_{s}$ is the number of samples. As expected, it can be seen from the inset of Fig. 2(c) that the sum of each component of the net dipole moment approaches zero when the number of samples increases.

The net dipole increases quickly up to $4 \%$, and then it decreases slowly. The latter is due to the presence of many nearest $\mathrm{N}-\mathrm{C}$ bonds which can cancel each other and cause a reduction in the net dipole. We conclude that the maximum dipole in the system occurs at around 4\% random doping. This is very promising for using the ferroelectricity effect of small $\mathrm{N}$-doped graphene samples. The induced polarization in the system strongly affects the electronic transport in the system [14]. The strength and direction of the net dipole can be controlled by positioning the nitrogen atoms on predefined locations. This will allow one to engineer the piezoelectric response of a NG sheet, which under applied stress leads to possible technological applications, such as the conversion of mechanical stimuli into an electrical signal and vice versa [26].

\section{B. Structural deformations}

The height variation at the position of the dopants is in good agreement with recent experimental studies. The height variations of single, double, and more complex dopant configurations were measured by using experimental and simulated scanning tunneling microscopy (STM) imaging $[7,10]$. In Fig. 3(a) we show a typical height distribution of doped graphene with $5 \%$ nitrogen concentration as obtained from our MD simulation. Figure 3(b) shows the height profile across two typical N clusters. Carbon (nitrogen) atoms are shown as the blue (brown) balls. Note that local ripples appear when $\mathrm{N}$ atoms form a cluster (adjacent brown balls). Here, the NG sheet was simulated at room temperature, as in recent experiments. Our results for the average peak height for a single dopant $(\sim 1.0 \AA)$ is in good agreement with the STM images in Ref. [18]. It was found experimentally that the apparent maximum out-of-plane height of the $\mathrm{N}$ dopant varies between 0.4 and $0.8 \AA$, and this is consistent with an $\mathrm{N}$ substitution for a $\mathrm{C}$ atom in the plane of graphene.

We also studied the effect of $\mathrm{N}$ dopants on the length of the C-C, C-N, and N-N bonds. This helps us to figure out the strength corresponding to each bond. In Fig. 4(b) we show the density plot of the bond length distribution at $0 \mathrm{~K}$. For pristine graphene the $\mathrm{C}-\mathrm{C}$ bond length is $1.43 \AA$ while in the presence of $5 \% \mathrm{~N}$ concentration the $\mathrm{C}-\mathrm{N}$ bond length is found to be $1.37 \AA$ (dark spots). The N-N bond has an average length of $1.57 \AA$ (bright spots). Our results are in good agreement with recent $a b$ initio calculations $[15,16]$ where they reported $\mathrm{C}-\mathrm{N}$ and $\mathrm{N}-\mathrm{N}$ bond lengths of 1.30 and $1.60 \AA$, respectively. Note that the bond length slightly depends on the dopant configuration, which is the reason why we give the average $\mathrm{C}-\mathrm{N}$ and $\mathrm{N}-\mathrm{N}$ bond lengths for different possible $\mathrm{N}$ configurations.

\section{The formation energy}

The formation energy $E_{f}$ is an interesting quantity to measure the stability of a system. For doped graphene it is given by

$$
E_{f}=\left[E_{\mathrm{NG}}-n_{\mathrm{C}} \varepsilon_{\mathrm{C}}-n_{\mathrm{N}} \varepsilon_{\mathrm{N}}\right] / n_{\mathrm{N}}
$$

where $E_{\mathrm{NG}}$ is the total energy of the studied system, $n_{\mathrm{C}}\left(n_{\mathrm{N}}\right)$ is the number of carbon (nitrogen) atoms, and $\varepsilon_{\mathrm{C}}(-8.39 \mathrm{eV})$ and $\varepsilon_{\mathrm{N}}(-4.77 \mathrm{eV})$ are, respectively, ReaxFF calculated atomic binding energies for pristine graphene and a free $\mathrm{N}_{2}$ molecule as reference systems [15,27]. 


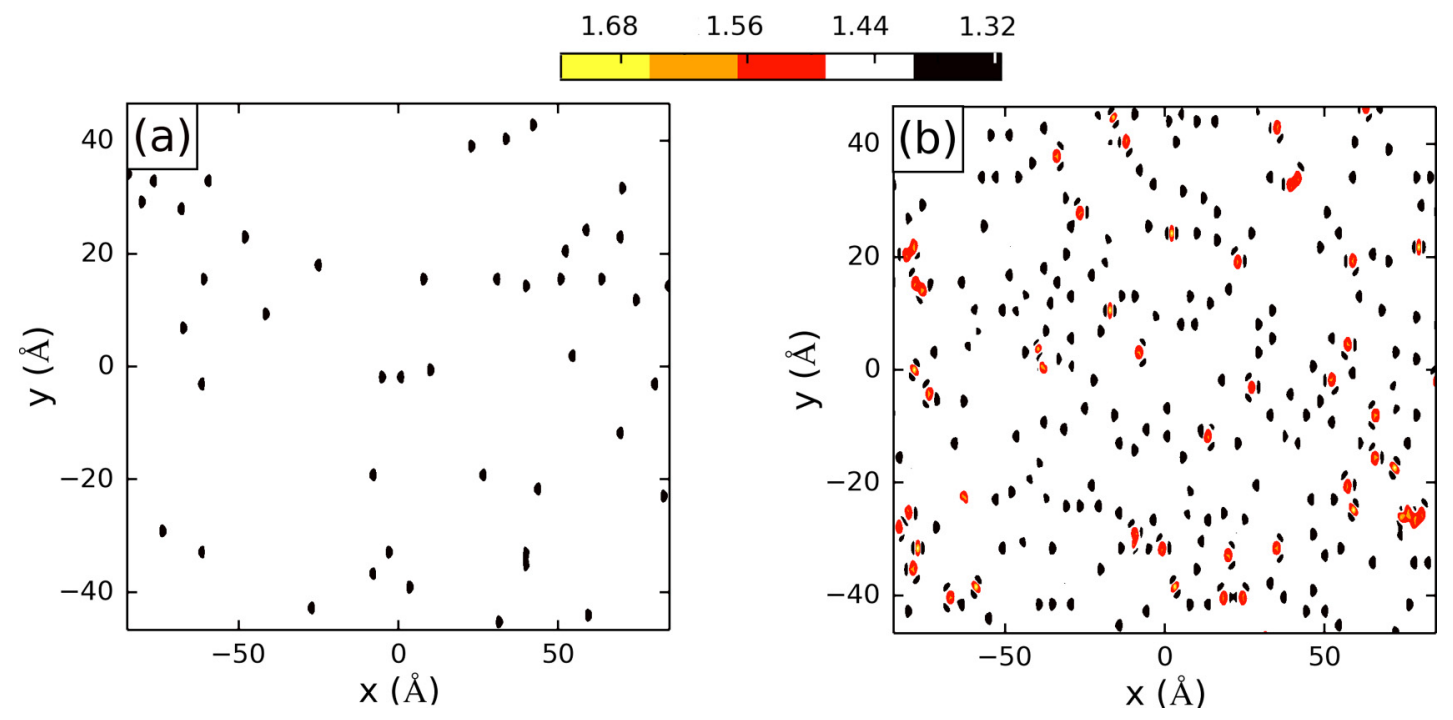

FIG. 4. Bond length distribution for the N-doping concentrations (a) $1 \%$ and (b) $5 \%$. Dark spots show a single nitrogen atom where the $\mathrm{C}-\mathrm{N}$ bond length becomes shorter. Bright spots indicate the N-N bond length which is longer than C-C (1.42 $⿱$ A). The scale is in $\AA$.

Energy per atom $E_{\mathrm{C}}\left(E_{\mathrm{N}}\right)$ is the average total energy of all carbon (nitrogen) atoms for a specific $\mathrm{N}$-doping concentration:

$$
\begin{aligned}
& E_{\mathrm{C}}(p)=\left[\sum_{i=1}^{n_{\mathrm{C}}} E_{i}^{\mathrm{tot}}(p)\right] / n_{\mathrm{C}}, \\
& E_{\mathrm{N}}(p)=\left[\sum_{i=1}^{n_{\mathrm{N}}} E_{i}^{\mathrm{tot}}(p)\right] / n_{\mathrm{N}},
\end{aligned}
$$

$n_{\mathrm{N}}=p N, n_{\mathrm{C}}+n_{\mathrm{N}}=N$, and $E_{i}^{\text {tot }}$ is the energy of the $i$ th atom. In ReaxFF, $E_{i}^{\text {tot }}$ contains many terms such as bond order, Coulomb, van der Waals, etc. The major contributions for carbon atoms are due to the bond order term (for more details, see Ref. [19]). The larger the absolute value of $E_{C}$ and $E_{N}$, the closer are the carbon atoms and nitrogen atoms to their ground state energy as compared to one carbon atom in pristine graphene and $\mathrm{N}_{2}$ molecules, respectively.

We present in Fig. 5(a) the formation energy of NG systems as a function of dopant concentration. Notice that the formation energy increases nonlinearly with dopant concentration. This confirms that $\mathrm{N}$-doped graphene is less stable than pristine graphene. At low doping concentrations (i.e., $p<1 \%$ ) $E_{f}$ rapidly increases due to single substitution of dopants. For higher concentrations (i.e., $p>3.0 \%$ ) the doped system becomes predominantly saturated by $\mathrm{N}$ clusters and then $E_{f}$ increases more. Our result is qualitatively in agreement with the $a b$ initio calculations for small size systems [7]. In a recent study, the formation energy of different $\mathrm{N}$ doping configurations was computed and it was found that each configuration reduces the system stability for single $(0.2 \mathrm{eV})$, double (0.5-1.7 eV), and more complex (1.9-11.6 eV) substitutions of nitrogen dopants. We also calculated the energy per atom for carbon $\left(E_{\mathrm{C}}\right)$ and nitrogen $\left(E_{\mathrm{N}}\right)$ for different NG systems. The results are shown in Fig. 5(b). It is seen that the binding energy of nitrogen atoms increases linearly with the number of dopants, which is due to the sharing of the electrons of nitrogen atoms with several carbon atoms. However, the binding energies of the carbon atoms lose less energy as compared to those of the nitrogen atoms.
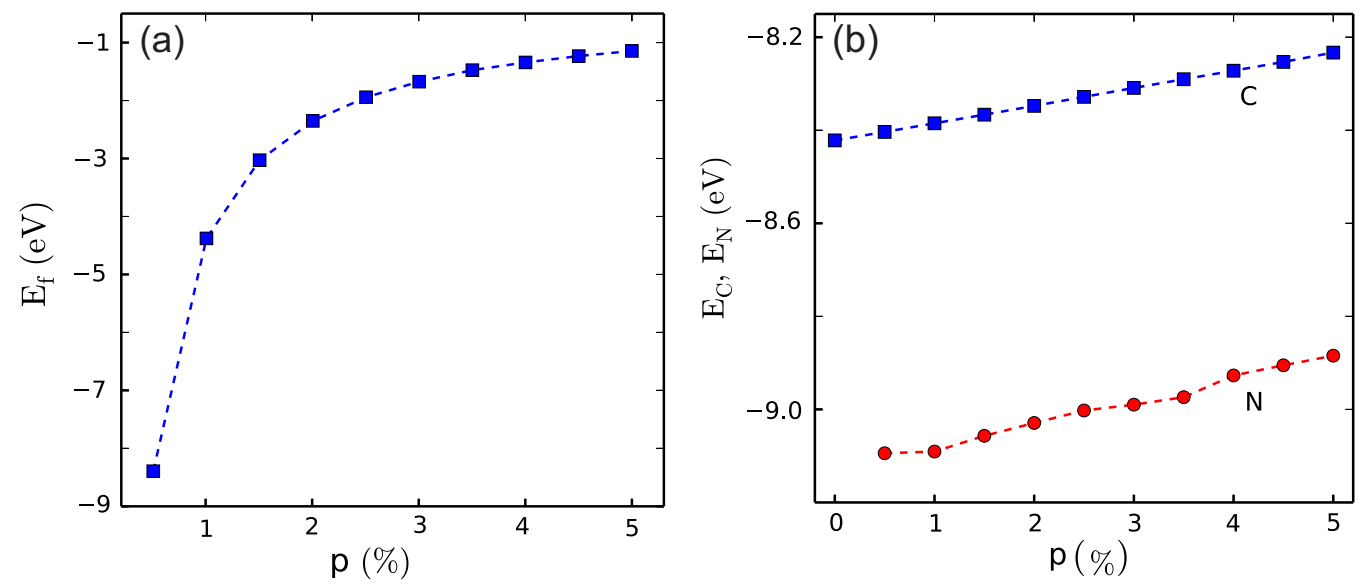

FIG. 5. (a) The formation energy $E_{f}$ and (b) energy per atom for carbon $E_{\mathrm{C}}$ and nitrogen $E_{\mathrm{N}}$ as a function of doping concentration at zero temperature. 

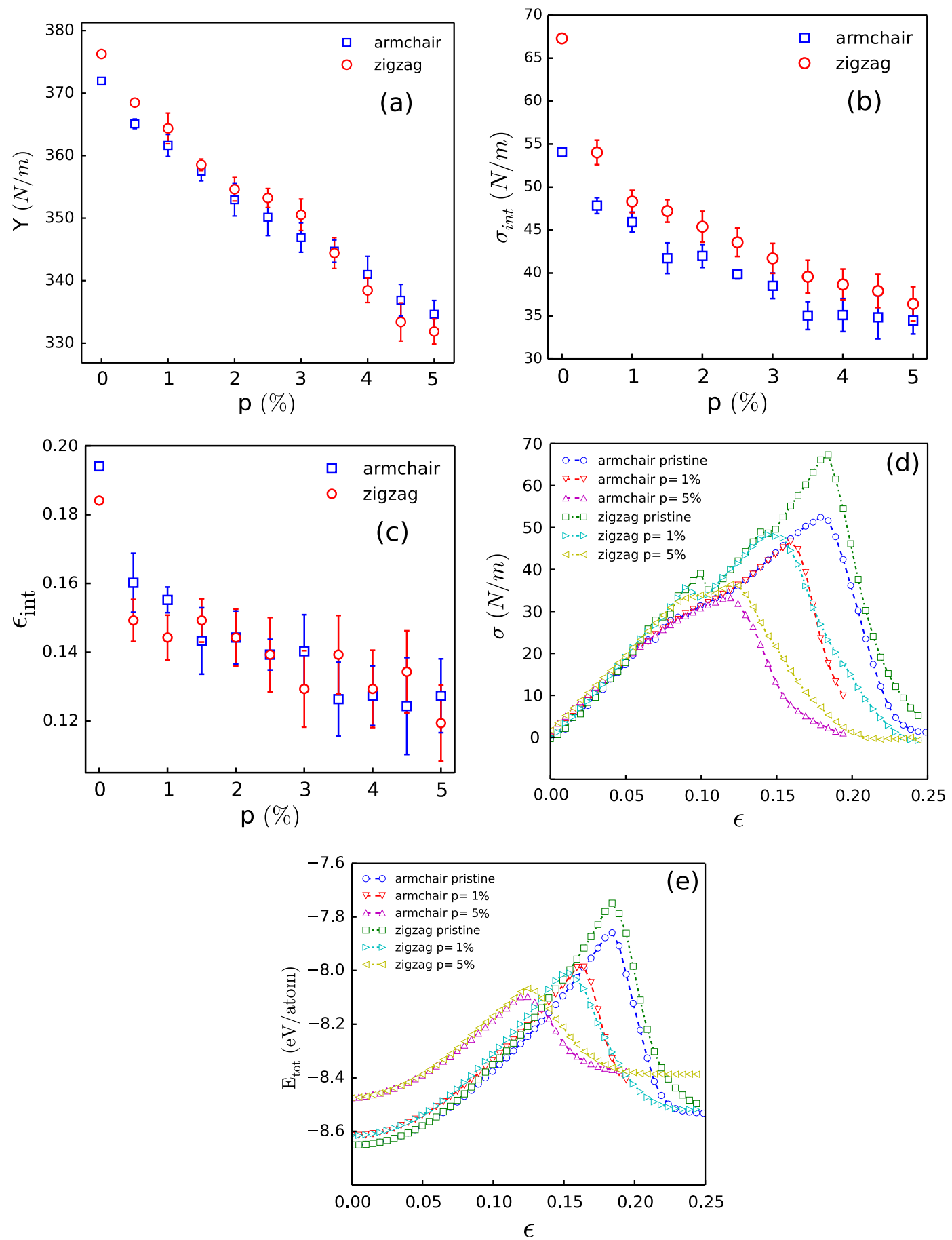

FIG. 6. (a) The Young's modulus $Y$, (b) breaking stress $\sigma_{\text {int }}$, and (c) breaking strain $\epsilon_{\text {int }}$ as a function of the N-dopant concentration $p$ in both armchair $(x)$ and zigzag $(y)$ directions. The stress-strain curve and the total energy for different systems: pristine, $1 \%$, and 5\% NG vs applied strain along $x(y)$ are shown respectively in (e) and (d). $\sigma$ and $\epsilon$ are the applied stress and strain.

\section{The Young's modulus and the breaking of doped graphene}

The mechanical properties of graphene have been intensively investigated using simulations at the atomic level. These studies obtained various elastic properties including Young's modulus $Y$, Poisson's ratio $v$, and breaking (intrinsic) strength $\sigma_{\text {int }}$ and strain $\epsilon_{\text {int }}$. Both DFT and tight binding approaches were used [18,27-29]. Moreover, experiments used a nanoindentation technique to measure the linear and nonlinear elasticity of free-standing pristine graphene [30].
Here, we study the effects of substitutional nitrogen doping on the Young's modulus, and intrinsic stress and strain of a graphene monolayer. We found that $\mathrm{N}$ doping decreases the Young's modulus [see Fig. 6(a)]. The result for pristine graphene $Y_{0}\left(369 \mathrm{~N} \mathrm{~m}^{-1}\right)$ is in good agreement with the reported experimental [30] value $340 \pm 50 \mathrm{~N} \mathrm{~m}^{-1}$. The reason for the weakening of NG is the nonuniformity of the bond length distribution. The longer the bond length and the nonuniform distribution of the bond lengths causes a more 
TABLE I. Young's modulus $(Y)$, breaking stress $\left(\sigma_{\text {int }}\right)$. and breaking strain $\left(\epsilon_{\text {int }}\right)$ in doped graphene for different concentrations of nitrogen atoms. The NG sheet is subjected to stress in the armchair $(x x)$ and zigzag $(y y)$ direction. $Y_{0}$ is the Young's modulus of pristine graphene.

\begin{tabular}{lcccc}
\hline \hline$p(\%)$ & $Y^{x x}(\mathrm{~N} / \mathrm{m})$ & $Y^{x x} / Y_{0}$ & $\sigma_{\text {int }}^{x x}(\mathrm{~N} / \mathrm{m})$ & $\epsilon_{\text {int }}^{x x}(\%)$ \\
\hline Ref. [30] & $340 \pm 50$ & & $42 \pm 4$ & 25.0 \\
Pristine & 372 & 1.00 & 54 & 19.4 \\
1 & 362 & 0.97 & 46 & 15.5 \\
2 & 353 & 0.94 & 42 & 14.4 \\
3 & 347 & 0.93 & 39 & 14.0 \\
4 & 341 & 0.91 & 35 & 12.7 \\
5 & 335 & 0.90 & 34 & 12.7 \\
$p(\%)$ & $Y^{y y}(\mathrm{~N} / \mathrm{m})$ & $Y^{y y} / Y_{0}$ & $\sigma_{\text {int }}^{y y}(\mathrm{~N} / \mathrm{m})$ & $\epsilon_{\text {int }}^{y y}(\%)$ \\
\hline Ref. [30] & $340 \pm 50$ & & $42 \pm 4$ & 25.0 \\
Pristine & 376 & 1.00 & 67 & 18.4 \\
1 & 364 & 0.97 & 48 & 14.4 \\
2 & 354 & 0.94 & 45 & 14.4 \\
3 & 350 & 0.93 & 41 & 12.9 \\
4 & 338 & 0.90 & 38 & 12.9 \\
5 & 331 & 0.88 & 36 & 11.9 \\
\hline \hline
\end{tabular}

local rippling of the position where the dopants are inserted. The decrease in the Young's modulus was also reported by Mortazavi et al. [17] where they used an optimized Tersoff potential [31], however, our results show a very smooth linear decrease which is different than what was found in Ref. [17]. It is also important to note that the optimized Tersoff potential yields a barely noticeable reduction in the Young's modulus of graphene with 5\% nitrogen doping. Our results, however, indicate a $10 \%$ reduction in $Y$. Probably this difference is due to the use of the Tersoff potential which is nonreactive and less flexible than the ReaxFF potential. In Table I we list a set of elastic constants for different $\mathrm{N}$-dopant concentrations ( $p$ ) and we compare the results with the experimental result for pristine graphene. In Figs. 6(b) and 6(c), $\sigma_{\text {int }}$ and $\epsilon_{\text {int }}$ are found to decrease with the number of dopants. We report a significant drop at low concentrations (i.e., $p<1 \%$ ), while at high concentrations (i.e., $p>3 \%$ ) these parameters slowly decrease due to weak N-N bonds inside the $\mathrm{N}$ clusters. The exponentially decreasing behavior is more favorable than a linear one because, by increasing the $\mathrm{N}$ concentration, the number of C-N bonds (and N-N bonds) increases and there is an increased chance to have two $\mathrm{C}-\mathrm{N}$ bonds adjacent to each other. The latter results in a nonlinear increasing disturbance in the C-N, C-C, and N-N bond lengths. Note that the breaking stress (strain) is defined as its maximum stress reached before breaking occurs. We found the intrinsic stress (strain) for pristine graphene to be $54 \mathrm{~N} \mathrm{~m}^{-1}(19.4 \%)$ in the armchair direction and $67 \mathrm{~N} \mathrm{~m}^{-1}$ (18.4\%) in the zigzag direction, which is larger (smaller) than the measured value $42 \pm 4 \mathrm{~N} \mathrm{~m}^{-1}$ (25) in Refs. [30,32]. The reason for this discrepancy might be due to the presence of defects and impurities in the experimental samples. A typical stress-strain curve is shown in Fig. 6(d). It is seen that pristine graphene has a larger breaking stress and strain as compared to its $1.5 \%$ nitrogen-doped counterpart. It is also interesting to note that the potential energy has the same quadratic increase versus strain in both the armchair and zigzag directions before the breaking point, which indicates that the NG samples obey linear elasticity [see Fig. 6(e)]. Notice that the Young's modulus of the graphene sheet was found to be almost independent of the loading direction [see Fig. 6(a)], while the intrinsic stress and strain, as shown in Figs. 6(b) and $6(\mathrm{c})$, depend on the direction of the applied force. This is in agreement with previous reports by Mortazavi et al. [17] and Zhao et al. [18]. In fact, graphene is isotropic from the point of view of linear elasticity but anisotropic from the point of view of nonlinear elasticity. This results from group theory applied to the graphene crystal structure [33]. For example, the total energy shown in Fig. 6(e) for pristine graphene is the same for applied stress along the zigzag and the armchair directions for strains smaller than $10 \%$ while they start to be different beyond this amount of strain where nonlinear effects become important.

In a recent study, Mortazavi et al. [17] used an optimized version of the Tersoff potential to study the mechanical properties of defected and doped graphene nanoribbons. They found that $Y$ was independent of the $\mathrm{N}$ concentration. In contrast, we found a linear decrease of $Y$ with $\mathrm{N}$ concentration, which we believe is correct. This discrepancy can be due to the nonreactive $\mathrm{N}-\mathrm{C}$ bonds (and nearest $\mathrm{C}-\mathrm{C}$ bonds) in their simulations, which cause a different $\mathrm{N}-\mathrm{C}$ bond length distribution and prevent the weakening of the sheet. On the other hand, our calculated breaking stress (strain) is in agreement with their results, i.e., $52.7 \mathrm{~N} / \mathrm{m}(0.23)$ [17].

\section{CONCLUSIONS}

In summary, using molecular dynamics simulations, the structural and mechanical properties of nitrogen-doped graphene were studied. We found that the roughness of the NG systems is sensitive to the dopant concentration. The major contribution to the ripples is from the nitrogen cluster formation in the graphene layer. The graphene layer is mechanically weaker when doped with nitrogen, resulting in a decrease of the Young's modulus and the breaking stress. The formation energy increases as a function of the number of dopants, which results in a decrease in the stability of the nitrogen-doped graphene.

\section{ACKNOWLEDGMENT}

This work was supported by the ESF-Eurographene project CONGRAN, and the Flemish Science Foundation (FWO-V1).
[1] K. S. Novoselov, A. K. Geim, S. V. Morozov, D. Jiang, Y. Zhang, S. V. Dubonos, I. V. Grigorieva, and A. A. Firsov, Science 306, 666 (2004).

[2] A. K. Geim and K. S. Novoselov, Nat. Mater. 6, 183 (2007).

[3] A. K. Geim, Science 324, 1530 (2009).
[4] C. Xu, H. Li, and K. Banerjee, IEEE Trans. Electron Devices 55, 3264 (2008).

[5] T. J. Booth, P. Blake, R. R. Nair, D. Jiang, E. W. Hill, U. Bangert, A. Bleloch, M. Gass, K. S. Novoselov, M. I. Katsnelson, and A. K. Geim, Nano Lett. 8, 2442 (2008). 
[6] F. Wang, Y. B. Zhang, C. S. Tian, C. Girit, A. Zettl, M. Crommie, and Y. R. Shen, Science 320, 206 (2008).

[7] R. Lv, Q. Li, A. R. Botello-Méndez, T. Hayashi, B. Wang, A. Berkdemir, Q. Hao, A. L. Elías, R. Cruz-Silva, and H. R. Gutiérrez, Sci. Rep. 2, 586 (2012).

[8] D. Geng, S. Yang, Y. Zhang, J. Yang, J. Liu, R. Li, T. Sham, X. Sun, S. Ye, and S. Knights, Appl. Surf. Sci. 257, 9193 (2011).

[9] Y. Lu, S. Lo, J. Lin, W. Zhang, J. Lu, F. Liu, C. Tseng, Y. Lee, C. Liang, and L. Li, ACS Nano 7, 6522 (2013).

[10] L. Zhao, R. He, K. T. Rim, T. Schiros, K. S. Kim, H. Zhou, C. Gutiérrez, S. P. Chockalingam, C. J. Arguello, and L. Pálová, Science 333, 999 (2011).

[11] H. Wang, T. Maiyalagan, and X. Wang, ACS Catal. 2, 781 (2012).

[12] T. X. Cui, R. Lv, Z. H. Huang, H. Zhu, J. Zhang, Z. Li, Y. Jia, F. Kang, K. Wang, and D. Wu, Carbon 49, 5022 (2011).

[13] R. T. Lv, T. X. Cui, M. S. Jun, Q. Zhang, A. Cao, D. S. Su, Z. Zhang, S. H. Yoon, J. Miyawaki, and I. Mochida, Adv. Funct. Mater. 21, 999 (2011).

[14] H. Liu, Y. Liu, and D. J. Zhu, J. Mater. Chem. 21, 3335 (2011).

[15] Y. Fujimoto and S. Saito, Phys. Rev. B 84, 245446 (2011).

[16] P. Rani and V. K. Jindal, RSC Adv. 3, 802 (2013).

[17] B. Mortazavi, S. Ahzi, V. Toniazzo, and Y. Rémond, Phys. Lett. A 376, 1146 (2012).

[18] H. Zhao, K. Min, and N. R. Aluru, Nano Lett. 9, 3012 (2009).
[19] A. C. T. Van Duin, S. Dasgupta, F. Lorant, and W. A. Goddard, J. Phys. Chem. A 105, 9396 (2001).

[20] J. Budzien, A. P. Thompson, and S. V. Zybin, J. Phys. Chem. 113, 13142 (2009).

[21] S. Nosé, J. Chem. Phys. 81, 511 (1984).

[22] W. G. Hoover, Phys. Rev. A 31, 1695 (1985).

[23] S. Plimpton, J. Comput. Phys. 117, 1 (1995).

[24] H. M. Aktulga, J. C. Fogarty, S. A. Pandit, and A. Y. Grama, Parallel Comput. 38, 245 (2012).

[25] A. Zandiatashbar, G. H. Lee, S. J. An, S. Lee, N. Mathew, M. Terrones, T. Hayashi, C. R. Picu, J. Hone, and N. Koratkar, Nat. Commun. 5, 3186 (2014).

[26] M. Zelisko, Y. Hanlumyuang, S. Yang, Y. Liu, C. Lei, J. Li, P. M. Ajayan, and P. Sharma, Nat. Commun. 5, 4284 (2014).

[27] K. N. Kudin, G. E. Scuseria, and B. I. Yakobson, Phys. Rev. B 64, 235406 (2001)

[28] M. Sammalkorpi, A. Krasheninnikov, A. Kuronen, K. Nordlund, and K. Kaski, Phys. Rev. B 70, 245416 (2004).

[29] G. Cao, Polymers 6, 2404 (2014).

[30] C. Lee, X. Wei, J. W. Kysar, and J. Hone, Science 321, 385 (2008).

[31] L. Lindsay and D. A. Broido, Phys. Rev. B 81, 205441 (2010).

[32] E. Cadelano, P. L. Palla, S. Giordano, and L. Colombo, Phys. Rev. Lett. 102, 235502 (2009).

[33] L. Colombo and S. Giordano, Rep. Prog. Phys. 74, 116501 (2011). 